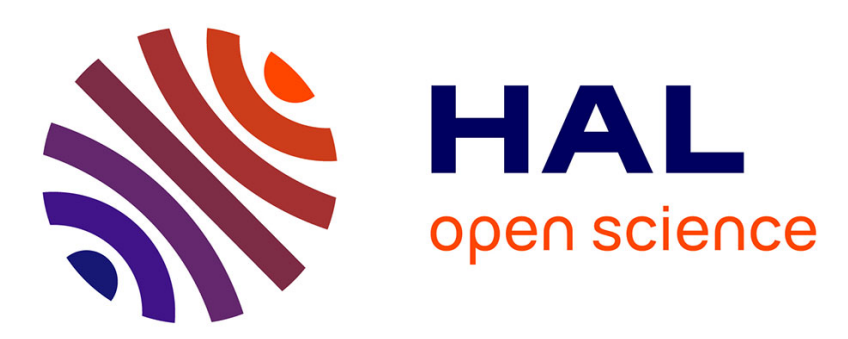

\title{
Cancer broncho-pulmonaire et exposition professionnelle aux fumées de soudage
}

Isabelle Stücker, Mireille Matrat

\section{To cite this version:}

Isabelle Stücker, Mireille Matrat. Cancer broncho-pulmonaire et exposition professionnelle aux fumées de soudage. Les cahiers de la Recherche. Santé, Environnement, Travail, 2013, Les risques sanitaires liés aux milieux aériens et hydriques, 2, pp.21-22. anses-01709933

\section{HAL Id: anses-01709933}

\section{https://hal-anses.archives-ouvertes.fr/anses-01709933}

Submitted on 15 Feb 2018

HAL is a multi-disciplinary open access archive for the deposit and dissemination of scientific research documents, whether they are published or not. The documents may come from teaching and research institutions in France or abroad, or from public or private research centers.
L'archive ouverte pluridisciplinaire HAL, est destinée au dépôt et à la diffusion de documents scientifiques de niveau recherche, publiés ou non, émanant des établissements d'enseignement et de recherche français ou étrangers, des laboratoires publics ou privés. 


\section{Cancer broncho-pulmonaire et exposition professionnelle aux fumées de soudage}

Cancer broncho-pulmonaire et exposition professionnelle aux fumées de soudage

Isabelle STUCKER et Mireille MATRAT

Mots-clés : cancer, poumon, soudage, expositions professionnelles

Le cancer broncho-pulmonaire (CBP) est la première cause de décès par cancer en France. Ainsi, en France, en 2005, on a dénombré 30.651 nouveaux cas (dont $78 \%$ chez l'homme) qui ont conduit à 26.624 décès. Le tabac est sans aucun doute le facteur de risque majeur du cancer du poumon. Cependant, les cancers des voies respiratoires sont particulièrement concernés par les expositions professionnelles, car l'inhalation est la voie de pénétration de nombreuses substances présentes en milieu de travail. Le cancer du poumon constitue d'ailleurs le cancer le plus lié aux expositions professionnelles ${ }^{40}$. Une publication récente à partir de l'étude Icare montre que près de $15 \%$ des cancers du poumon sont attribuables à une exposition professionnelle à des cancérogènes pulmonaires reconnus ${ }^{41}$.

\section{Le soudage}

Le soudage est une activité qui se rencontre dans une multitude de secteurs professionnels tels que la sidérurgie, le travail des métaux, la fabrication de machines et d'équipements, de matériels de transport, d'appareils électriques, d'instruments médicaux de précision, d'optique et d'horlogerie. En 2003, l'enquête Sumer ${ }^{42}$ estimait à 360.000 le nombre de personnes en France concernées par cette activité.

Le soudage est une opération d'assemblage de pièces, en général en métal, avec ou sans apport de métal supplémentaire, selon la technique utilisée. Diverses méthodes sont utilisées, pour provoquer la fusion du métal. On distingue ainsi :
- Le soudage à l'arc, le plus répandu, qui utilise la chaleur d'un arc électrique ;

- Le soudage au chalumeau qui utilise une flamme obtenue à partir d'un gaz (oxyacétylène ou propane) et l'oxygène ;

- Le soudage par pression

- Et d'autres types de soudages tels que

o Le soudage par faisceau d'électrons dont l'énergie cinétique se transforme en chaleur ;

o Le soudage laser où l'énergie est apportée sous la forme d'un faisceau laser, etc.

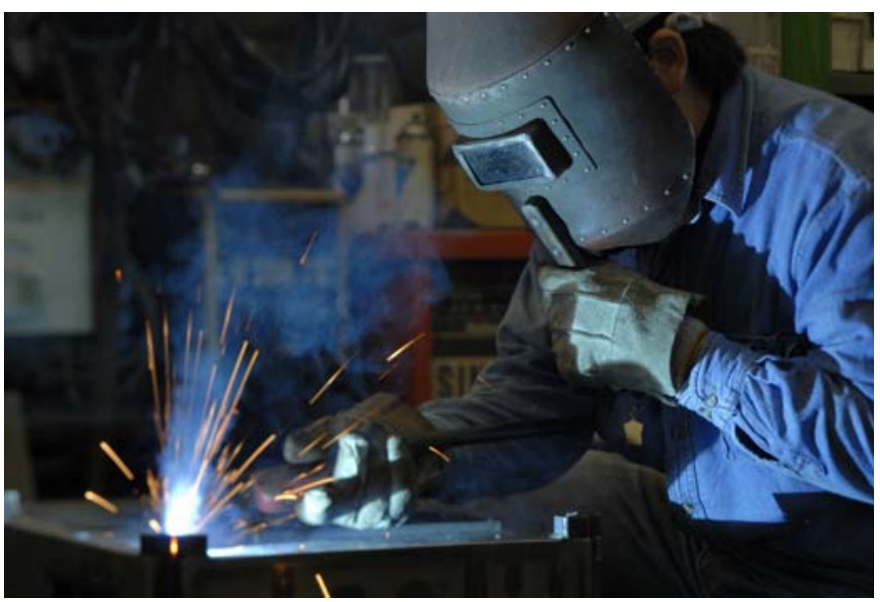

Les émissions polluantes dépendent du type de soudage et en particulier, des températures atteintes localement, des spécificités du procédé comme la composition du gaz et des métaux en présence, du revêtement ou des contaminants du métal soudé ce qui fait des fumées de

\section{Inserm UM 1018 Équipe 6 - Villejuif}

\footnotetext{
${ }^{40}$ Steenland K, Loomis D, Shy C, et al. Review of occupational lung carcinogens. Am J Ind Med 1996;29:474-90.2.

Siemiatycki J, Richardson L, Straif K, et al. Listing occupational carcinogens. Environ Health Perspect 2004;112:1447-59.

${ }^{41}$ Guida F, Papadopoulos A, Menvielle G et al. Risk of lung cancer and occupational history: results of a French population-based case-control study, the ICARE study. J Occup Environ Med 2011 ; 53 : 1068-77

${ }^{42}$ Surveillance médicale des risques professionnels: enquête mise en place par le Ministère du Travail, lancée et gérée conjointement par la Direction des Relations du Travail (Inspection médicale du travail) et la Direction de I'Animation de la Recherche, des Etudes et des Statistiques : www.travail-emploi.gouv.fr
} 
soudage un mélange complexe. Ainsi, ces fumées émettent des nuisances gazeuses ou particulaires telles que l'ozone, le formol, de nombreux oxydes notamment métalliques (chromates, fer, nickel, etc) des particules notamment sous forme nanoparticulaire.

\section{L'inhalation des fumées de soudage}

L'exposition aux particules métalliques diffère selon le type de matériaux soudé. Une exposition au chrome hexavalent (substance classée dans le groupe 1 par le Centre International sur le Cancer (CIRC), c'est à dire cancérogène certain chez l'homme) se rencontre particulièrement en cas de soudage manuel d'aciers inoxydables ou d'aciers faiblement alliés avec un métal d'apport inoxydable. D'autres substances peuvent également se rencontrer lors du soudage: nickel, fer, béryllium, cadmium... Les études épidémiologiques réalisées avant 1990 avaient conduit le CIRC à classer l'exposition aux fumées de soudage comme une activité possiblement cancérigène pour l'homme du fait de résultats discordants.

\section{Le projet de recherche : Icare-Soudure}

L'étude Icare est une étude cas-témoins ${ }^{43}$, mise en place pour étudier le rôle des facteurs de risque professionnels et environnementaux dans la survenue de cancers broncho-pulmonaires (CBP) et de cancers des voies aérodigestives supérieures. Dans ce projet, l'étude Icare a pour but de permettre :

- d'étudier en détail le rôle de l'exposition aux fumées de soudage dans la survenue de CBP, en fonction des caractéristiques de cette activité (type de soudure, technique de soudage, matériaux impliqués...) ;

- d'étudier les relations dose-effet en lien avec le temps passé à réaliser l'activité de soudage, le niveau d'exposition aux fumées, etc.

\section{Les partenaires :}

Isabelle Stucker

Inserm UM 1018 Équipe 6, Villejuif

Mireille Matrat

Centre Hospitalier Intercommunal de Créteil, Service de Pneumologie et Pathologie Professionnelle

Durée : 24 mois

Financement ITMO Cancer : $66.385 €$

Contact : isabelle.stucker@inserm.fr

${ }^{43}$ Design d'étude qui compare la proportion de personnes exposées à l'exposition d'intérêt entre un groupe de sujets malades de la pathologie d'intérêt et un groupe de sujets non malades de cette pathologie (le plus recommandé une population saine). 